\title{
Measuring central pulmonary pressures during exercise in COPD: how to cope with respiratory effects
}

\author{
Bart G. Boerrigter ${ }^{1}$, Aaron B. Waxman², Nico Westerhof ${ }^{1}$, \\ Anton Vonk-Noordegraaf ${ }^{1}$ and David M. Systrom ${ }^{2}$
}

Affiliations: 'Dept of Pulmonary Diseases, VU University Medical Center, Amsterdam, the Netherlands. ${ }^{2}$ Dept of Pulmonary and Critical Care Medicine, Harvard Medical School, Brigham and Women's Hospital, Boston, MA, USA.

Correspondence: A. Vonk-Noordegraaf, Dept of Pulmonary Diseases, VU University Medical Center, De Boelelaan 1117, 1081 HV Amsterdam, the Netherlands. E-mail: a.vonkavumc.nl

ABSTRACT Respiratory influences are major confounders when evaluating central haemodynamics during exercise. We studied four different methods to assess mean pulmonary artery pressure (mPAP) and pulmonary capillary wedge pressure (PCWP) in cases of respiratory swings.

Central haemodynamics were measured simultaneously with oesophageal pressure during exercise in 30 chronic obstructive pulmonary disease (COPD) patients. MPAP and PCWP were assessed at the end of expiration, averaged over the respiratory cycle and corrected for the right atrial pressure (RAP) waveform estimated intrathoracic pressure, and compared with the transmural pressures.

Bland-Altman analyses showed the best agreement of mPAP averaged over the respiratory cycle (bias (limits of agreement) $2.5(-6.0-11.8) \mathrm{mmHg})$ and when corrected with the nadir of RAP $(-3.6(-11.2-3.9)$ $\mathrm{mmHg})$. Measuring mPAP at the end of expiration $(10.3(0.5-20.3) \mathrm{mmHg})$ and mPAP corrected for the RAP swing $(-9.3(-19.8-2.1) \mathrm{mmHg})$ resulted in lower levels of agreement. The respiratory swings in mPAP and PCWP were similar $\left(\mathrm{r}^{2}=0.82\right.$, slope \pm SE $\left.0.95 \pm 0.1\right)$.

Central haemodynamics measured at the end of expiration leads to an overestimation of intravascular pressures in exercising COPD patients. Good measurement can be acquired even when oesopghageal pressure is omitted, by averaging pressures over the respiratory cycle or using the RAP waveform to correct for intrathoracic pressure. Assessment of the pulmonary gradient is unaffected by respiratory swings.

@ERSpublications

Measurement of intravascular pressures in exercising COPD patients requires correction for intrathoracic pressure http://ow.ly/tVLlg

Received: Jan 292013 | Accepted after revision: Oct 222013 | First published online: Oct 312013

Support statement: A. Vonk-Noordegraaf was supported by the Netherlands Organisation for Scientific Research Vidi (project number 917.96.306).

Conflict of interest: Disclosures can be found alongside the online version of this article at www.erj.ersjournals.com

Copyright @ERS 2014 


\section{Introduction}

Haemodynamic assessment remains the gold standard in the diagnosis of cardiovascular and pulmonary vascular disease. In an effort to detect early and potentially more treatable pulmonary vascular disease $[1,2]$, and heart failure with preserved ejection fraction [3], there has been renewed interest in the direct measurement of central pulmonary haemodynamics during exercise. Both heart and vessel abnormalities are frequently found in chronic obstructive pulmonary disease (COPD) [4, 5]. To minimise the effect of increased intrathoracic pressure changes on intravascular pressure measurements during right heart catheterisation at rest, it is recommended that pressures be analysed at the end of expiration [6]. During exercise in patients with COPD in particular, the increase in intrathoracic pressure during expiration can be a major confounder of the measurement of central pulmonary pressures.

COPD is characterised by a combination of derangements in respiratory mechanics, which alter intrathoracic pressure. At rest, increased lung compliance requires a larger lung volume to balance chest wall recoil. During exercise, increased compliance and resistance lead to ineffective and prolonged gas emptying on expiration. This causes inspiration to start before a full expiration has occurred, before the lung has returned to is relaxation volume. As a consequence, not only does the lung volume at the end of expiration rise dynamically (dynamic hyperinflation), the alveolar pressure at the end of expiration also becomes higher (intrinsic positive end-expiratory pressure $(\mathrm{PEEP} i)$ ). The consequence of this is that the respiratory muscles have to generate a more negative intrathoracic pressure during inspiration to overcome the presence of PEEPi, and, especially, generate a large positive intrathoracic pressure during expiration. These pressures are exaggerated in exercise. When measuring haemodynamics during exercise in COPD, it is preferable to subtract oesophageal pressure ( $\left.P_{\text {oes }}\right)$ in order to acquire the true intracavitary pressure, and thereby derive actual transmural pressure, which is critical in the detection of pulmonary vascular disease [7]. However, this is not practical in daily practice and, therefore, older studies reported the values of central pressures averaged over one or more respiratory cycles during exercise in COPD patients [8-10] and healthy subjects [11-13]. More recent studies abandoned this and reported end-expiratory measurements [14, 15], based on the recommendations for resting measurements under the assumption that they also hold during exercise. An alternative is to use the right atrial pressure (RAP) waveform, which is largely influenced by its surrounding pressure (which is intrathoracic pressure), to estimate intrathoracic pressure [16].

How to measure central pressures during exercise in cases of exaggerated intrathoracic pressure swings and the validity of using the RAP waveform to correct for intrathoracic pressure remain largely unknown. In this study, therefore, we evaluated the effect of intrathoracic pressure on the different central pressures in patients with COPD in order to find a method of adequately measuring pulmonary vascular pressures without measuring intrathoracic pressure in routine clinical practice. We hypothesised that measuring at the end of expiration leads to an overestimation of pulmonary vascular disease and that averaging over the respiratory cycle or correcting the pulmonary artery pressure (PAP) with an intrathoracic pressure results in better assessment of the intravascular pressure. Secondly, we hypothesise that the respiratory swings with exercise in PAP and wedge pressure are similar, so that the transpulmonary gradient can be assessed unaffected by respiratory swings.

\section{Methods}

Subjects

30 COPD patients were referred for the analysis of pulmonary hypertension. Patients were selected based on a moderate to very severe airway obstruction (forced expiratory volume in $1 \mathrm{~s}$ (FEV1) $<80 \%$ predicted) without significant reversibility $(<12 \%$ or $<200 \mathrm{~mL}$ change in FEV1 value). Patients were on optimal medical therapy and had no acute exacerbation for $\geqslant 4$ weeks. Pulmonary function testing was performed according to published guidelines $[17,18]$. All tests were performed at the VU University Medical Center (Amsterdam, the Netherlands) and the VU University Medical Center ethics committee approved the study. Written informed consent was obtained from all patients.

\section{Preparation of subjects}

A balloon-tipped, flow-directed, triple-lumen 7.5-F Swan-Ganz catheter (Baxter Healthcare Corporation, Irvine, CA, USA) was inserted under local anaesthesia into the jugular vein. The ports of the catheter were positioned in the right atrium, right ventricle and pulmonary artery. A radial artery catheter was inserted at either the right or left wrist under sterile conditions and following local anaesthesia. A standard oesophageal balloon catheter (Microtek Medical BV, Zutphen, the Netherlands) was inserted transnasally with the use of lidocaine gel (2\%). The balloon tip was first positioned $45 \mathrm{~cm}$ from the nares and the balloon was emptied by performing a Valsalva manoeuvre [19]. After this, $5 \mathrm{~mL}$ air was injected into the balloon followed by the withdrawal of $4 \mathrm{~mL}$, to partially inflate the balloon. The pressure signal was checked and, in case of cardiac artefacts, the position of the balloon catheter was slightly adjusted in the proximal direction. 
Patients were placed on an electromagnetically braked cycle ergometer (Ergoline GmbH, Bitz, Germany), in the semisupine position. The zero reference for the pressure transducers was $5 \mathrm{~cm}$ below the middle of the sternum. All catheters were connected to a PowerLab data acquisition system (ADInstruments, Dunedin, New Zealand) in order to record and digitalise pressures from the right atrium, right ventricle and pulmonary artery simultaneously with Poes.

\section{Protocol}

The protocol consisted of $3 \mathrm{~min}$ of rest after which workload was increased every 3 min until exhaustion. Oxygen consumption $\left(V^{\prime} \mathrm{O}_{2}\right)$ was measured continuously using a metabolic cart (Vmax 229; Viasys Healthcare, Yorba Linda, CA, USA). Patients were asked to signal just prior to exhaustion in case the last workload could not be fulfilled for $3 \mathrm{~min}$, to complete the data collection at maximal exercise. During the last $30 \mathrm{~s}$ of every workload, pulmonary capillary wedge pressure (PCWP) was acquired, and mixed venous and arterial blood samples were drawn. Cardiac output was calculated using the direct Fick method from arterial and mixed venous oxygen saturation and $V^{\prime} \mathrm{O}_{2}$. Pulmonary vascular resistance (PVR) was calculated as (mean PAP (mPAP) - PCWP)/cardiac output.

\section{Post-processing of pressure waveforms}

The recorded pressure tracings were visually checked and $30 \mathrm{~s}$ of good-quality pressure waveforms were selected near the end of each workload in addition to the part with the PCWP tracing. At each workload, we selected the PAP tracing as close as possible to the PCWP tracing to minimise changes in flow and intrathoracic pressure between the two measurements. Each selected timeframe was analysed using an inhouse programme for Matlab (MathWorks, Natick, MA, USA) using two different methods. We automatically acquired systolic, mean (mPAP) and diastolic PAP together with the transmural values of mPAP (mPAPtm) and PCWP (PCWPtm) by subtracting Poes in a beat-to-beat manner. All values were acquired and averaged over a period of 10-20 inspiratory and 10-20 expiratory heartbeats. This way, we acquired robust inspiratory and expiratory values separately as well as the swings (expiratory-inspiratory values) (fig. 1).

We then corrected expiratory MPAP and PCWP for the increased Poes in four different ways, and compared them with mPAPtm and PCWP, which we considered the gold standard. 1) End-expiratory: mPAP and PCWP measured at the moment of the last heartbeat during expiration without any further correction (mPAPend-exp and PCWPend-exp). 2) Averaged over the full respiratory cycle: mPAP and PCWP averaged over three full respiratory cycles including inspiratory and expiratory heartbeats (mPAPaveraged and PCWPaveraged). 3) Corrected for nadir RAP (RAPnadir): mPAP and PCWP at expiration corrected for the nadir in the RAP waveform during the same period of expiration. This assumes that during the relaxation period RAP falls to the pressure surrounding the right atrium and, therefore, is useful in estimating and correction for Poes [16] (mPAPRAP ${ }_{\text {nadir }}$ and PCWPRAP ${ }_{\text {nadir }}$ ). Figure 2 shows the determination of RAPnadir. 4) Corrected for RAP swing: mPAP and PCWP at expiration corrected for the swing in RAP from inspiration to expiration during the same breathing cycle. This assumes that the swing in RAP is useful in estimating and correction for Poes mPAPRAP swing $_{\text {and PCWP RAP }}$ swing. See figure 2 for the determination of the RAP swing.

\section{Statistical methods}

Demographic, pulmonary function and haemodynamic data are presented as mean \pm SD. Differences in the slope and absolute values at rest and exercise before and after correction were tested using a two-way ANOVA. The accuracy of the four different methods of measurements of mPAP and PCWP was evaluated by Bland-Altman analyses with mPAPtm and PCWPtm as the gold standards. Relationships were analysed by linear regression. Differences between respiratory swings at different pressures were tested using a twotailed t-test. $\mathrm{p}<0.05$ was considered significant. Analyses were performed using GraphPad Prism 5.0 (GraphPad, La Jolla, CA, USA).

\section{Results}

Study population

14 males and 16 females with a mean \pm SD age of $64 \pm 9$ years and a body mass index of $27 \pm 6 \mathrm{~kg} \cdot \mathrm{m}^{-2}$ were included in this study. Pulmonary function and haemodynamic measurements are summarised in table 1. All patients were former or current smokers (mean \pm SD $37 \pm 18$ pack-years), and had been diagnosed with emphysema based on pulmonary function and computed tomography of the chest. The severity of the airflow limitation was moderate in 16 patients, severe in 11 patients and very severe in three patients, according to the Global Initiative for Chronic Obstructive Lung Disease criteria. Nine patients were 

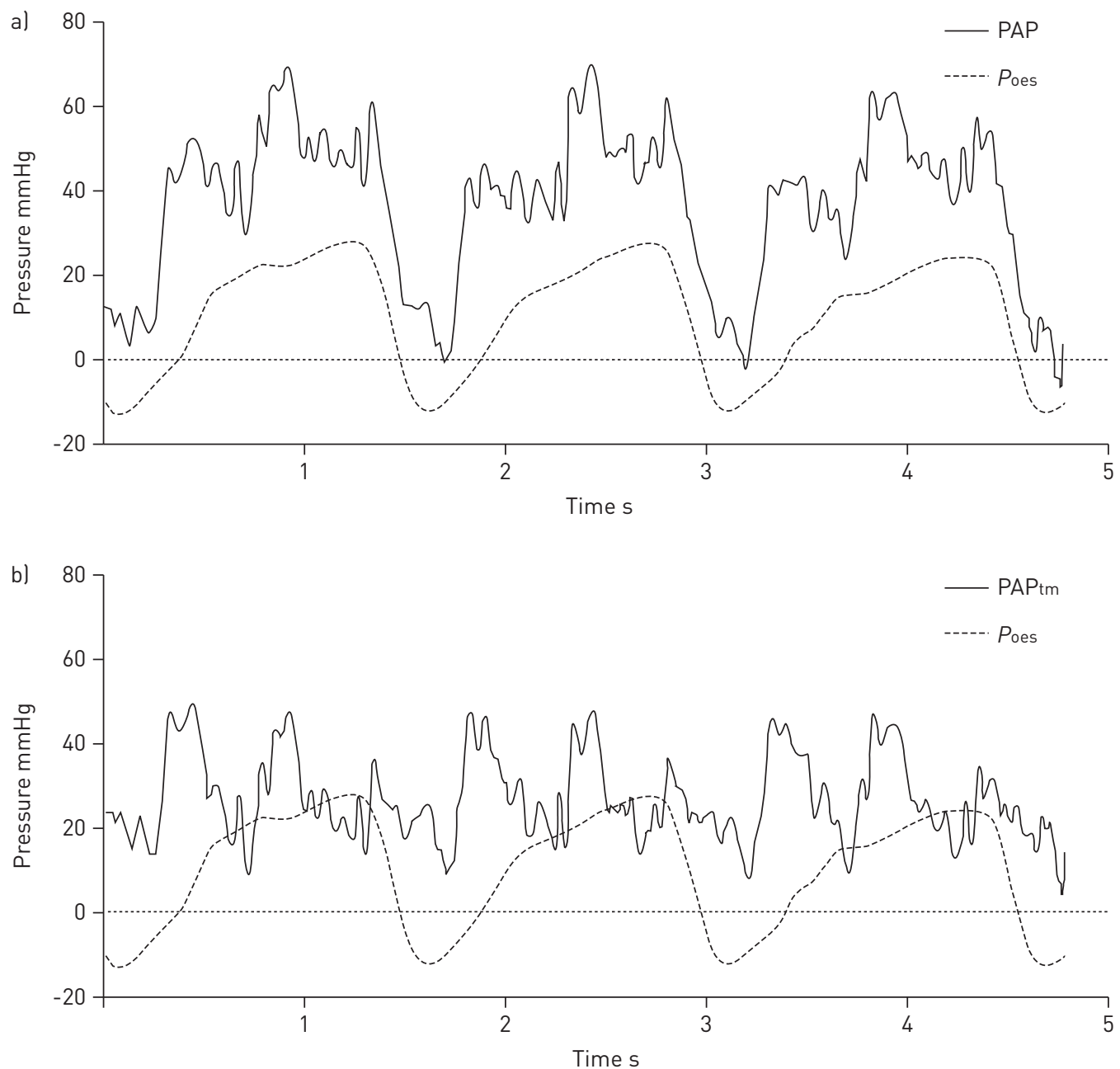

FIGURE 1 Example of pulmonary artery pressure (PAP) a) before and b) after (transmural PAP (PAPtm)) continuous correction for oesophageal pressure (Poes) at maximal exercise in a patient with severe chronic obstructive pulmonary disease (forced expiratory volume in $1 \mathrm{~s} \leqslant 30 \%$ predicted).

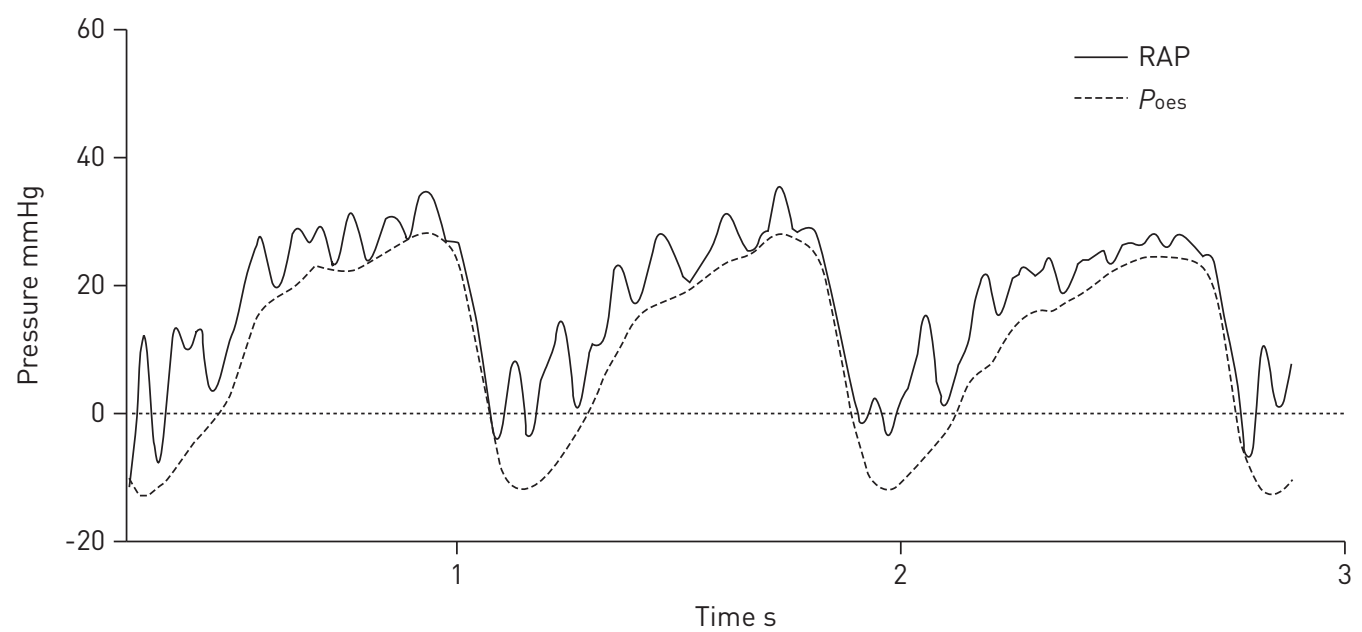

FIGURE 2 Example of determinations of right atrial pressure (RAP). The simultaneous measurement of RAP and oesophageal pressure (Poes) are shown at maximal exercise in the same patient as shown in figure 1. Nadir RAP is the lowest point in RAP during expiration, which represents RAP during relaxation. Note that RAP falls towards Poes during relaxation. RAP swing was determined as the difference between inspiratory RAP and expiratory RAP. 


\section{TABLE 1 Pulmonary function and haemodynamic characteristics}

\begin{tabular}{|c|c|}
\hline \multicolumn{2}{|l|}{ FEV 1} \\
\hline L & $1.58 \pm 0.62$ \\
\hline$\%$ pred & $56 \pm 18$ \\
\hline FEV $_{1} /$ VC \% & $46 \pm 18$ \\
\hline \multicolumn{2}{|l|}{ VC } \\
\hline $\mathrm{L}$ & $3.46 \pm 1.09$ \\
\hline$\%$ pred & $98 \pm 22$ \\
\hline \multicolumn{2}{|l|}{ TLC } \\
\hline L & $6.54 \pm 1.20$ \\
\hline$\%$ pred & $109 \pm 17$ \\
\hline \multicolumn{2}{|l|}{ FRC } \\
\hline $\mathrm{L}$ & $4.19 \pm 1.02$ \\
\hline$\%$ pred & $136 \pm 34$ \\
\hline Cardiac index $\mathrm{L} \cdot \mathrm{min}^{-1} \cdot \mathrm{m}^{-2}$ & $3.3 \pm 0.9$ \\
\hline Heart rate beats $\cdot \min ^{-1}$ & $80 \pm 17$ \\
\hline $\mathrm{PaO}_{2} \mathrm{mmHg}$ & $65 \pm 15$ \\
\hline $\mathrm{PaCO}_{2} \mathrm{mmHg}$ & $39 \pm 9$ \\
\hline
\end{tabular}

Data are presented as mean \pm SD. FEV1: forced expiratory volume in $1 \mathrm{~s} ; \%$ pred: \% predicted; VC: vital capacity; TLC: total lung capacity; FRC: functional residual capacity; $\mathrm{PaO}_{2}$ : arterial oxygen tension; $\mathrm{PaCO}_{2}$ : arterial carbon dioxide tension.

hypoxaemic (arterial oxygen tension $<60 \mathrm{mmHg}$ ) at rest while breathing room air and four patients had arterial carbon dioxide tension $>45 \mathrm{mmHg}$.

\section{Pressures during exercise with and without correction for intrathoracic pressure}

Maximal exercise was at a workload of $48 \pm 31 \mathrm{~W}$ with a $V^{\prime} \mathrm{O}_{2}$ of $922 \pm 379 \mathrm{~mL}$. A mean \pm SD cardiac output of $10.9 \pm 3.8 \mathrm{~L} \cdot \mathrm{min}^{-1}$ was reached with a heart rate of $118 \pm 19$ beats $\cdot \mathrm{min}^{-1}$. The central haemodynamic pressures are summarised in table 2. The large swings in Poes were transduced into all central pressure, on average responsible for a difference between inspiratory values and expiratory values of $\sim 20 \mathrm{mmHg}$. The expiratory Poes at maximal exercise ranged from $+3 \mathrm{mmHg}$ to $+25 \mathrm{mmHg}$ (fig. 3 ). The mean $\pm \mathrm{SD}$ slope decreased from $6.4 \pm 3.7 \mathrm{mmHg} \cdot \mathrm{L}^{-1}$ to $4.4 \pm 3.2 \mathrm{mmHg} \cdot \mathrm{L}^{-1}(\mathrm{p}<0.001)$ after correction for intrathoracic pressure. Seven patients had a $\mathrm{mPAP} /$ cardiac output slope $>3 \mathrm{mmHg}$ before correction, which decreased to $<3 \mathrm{mmHg}$ after correction for intrathoracic pressure. 19 out of the 22 patients who had a PCWP recording had a PCWP >20 mmHg with exercise without correction for intrathoracic pressure. Seven patients had a PCWPtm $>20 \mathrm{mmHg}$; in three of them PCWPtm was $20-25 \mathrm{mmHg}$.

\section{MPAP measurement and potential correction methods}

mPAPtm and PCWPtm at maximal exercise were $47 \pm 15 \mathrm{mmHg}$ and $17 \pm 8 \mathrm{mmHg}$, respectively. The average mPAP values at maximal exercise of each of the four methods were mPAPend-exp $59 \pm 14 \mathrm{mmHg}$, mPAPaveraged $50 \pm 14 \mathrm{mmHg}, \mathrm{mPAPRAP}_{\text {nadir }} 44 \pm 15 \mathrm{mmHg}$ and $\mathrm{mPAPRAP}_{\text {swing }} 38 \pm 15 \mathrm{mmHg}$. The average PCWP values at maximal exercise of each of the four methods were PCWPend-exp $27 \pm 9 \mathrm{mmHg}$, PCWPaveraged $20 \pm 8 \mathrm{mmHg} \mathrm{PCWPRAP}_{\text {nadir }}: 15 \pm 7 \mathrm{mmHg}$ and PCWPRAP swing $11 \pm 8 \mathrm{mmHg}$. Bland-Altman

TABLE 2 Central pressure over the respiratory cycle at rest and during exercise

\begin{tabular}{|c|c|c|c|c|c|c|}
\hline & \multicolumn{3}{|c|}{ Rest } & \multicolumn{3}{|c|}{ Exercise } \\
\hline & Expiration & Inspiration & Swing & Expiration & Inspiration & Swing \\
\hline sPAP mmHg & $53 \pm 20$ & $45 \pm 19$ & $8 \pm 6$ & $87 \pm 24$ & $64 \pm 21$ & $22 \pm 11$ \\
\hline mPAP $\mathrm{mmHg}$ & $33 \pm 12$ & $28 \pm 11$ & $6 \pm 6$ & $59 \pm 14$ & $40 \pm 13$ & $19 \pm 8$ \\
\hline dPAP $\mathrm{mmHg}$ & $23 \pm 9$ & $15 \pm 7$ & $8 \pm 5$ & $40 \pm 11$ & $23 \pm 11$ & $19 \pm 11$ \\
\hline
\end{tabular}

Data are presented as mean \pm SD. Poes: oesophageal pressure; s: systolic; PAP: pulmonary artery pressure; m: mean; d: diastolic; PCWP: pulmonary capillary wedge pressure; RAP: right atrial pressure. 

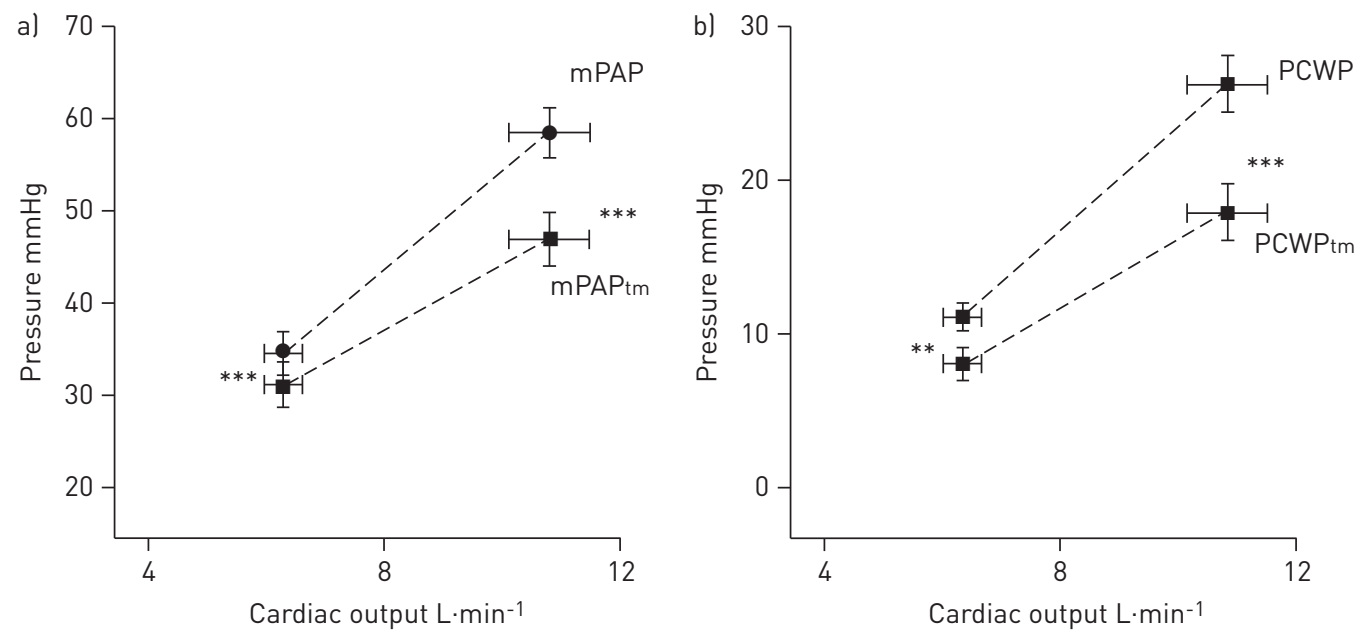

FIGURE 3 Average pressure flow relations before and after correction for oesophageal pressure (Poes). a) Mean pulmonary artery pressure $(\mathrm{mPAP})$ and transmural $\mathrm{mPAP}(\mathrm{mPAPtm})$ (calculated as $\mathrm{mPAP}-\mathrm{Poes}) ; \mathrm{b})$ pulmonary capillary wedge pressure (PCWP) and transmural PCWP (PCWPtm) (calculated as PCWP - Poes). ${ }^{* *}: \mathrm{p}<0.01 ;{ }^{* * *}: \mathrm{p}<0.001$.
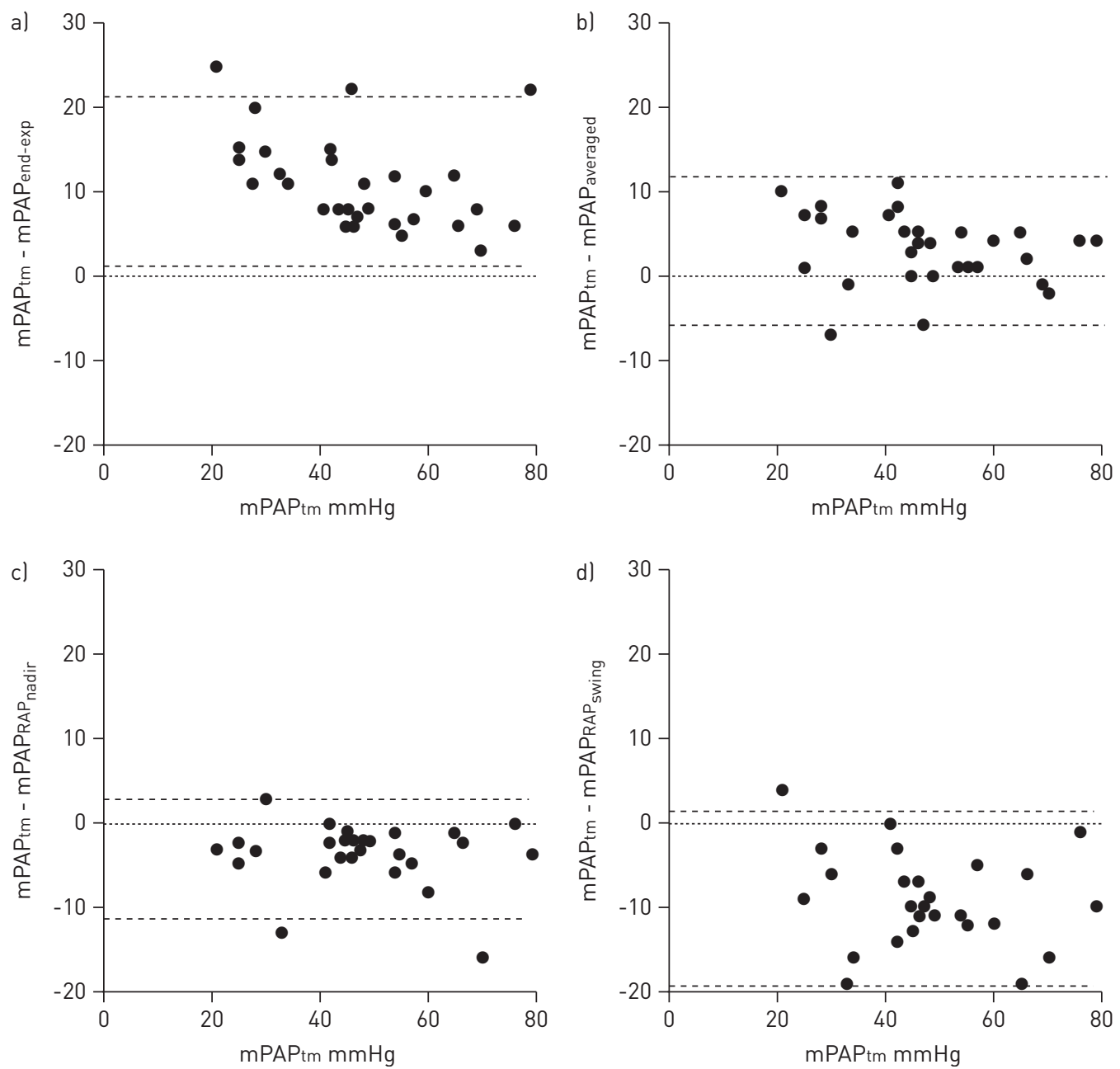

FIGURE 4 Bland-Altman analyses of the difference between mean pulmonary artery pressure (mPAP) and transmural mPAP (mPAPtm) plotted versus the mPAPtm. a) mPAP measured at the end of expiration (mPAPend-exp); b) mPAP averaged over the respiratory cycle (mPAPaveraged); c) mPAP corrected for the lowest point of right atrial pressure (RAP)

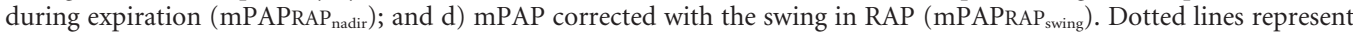
the $95 \%$ limits of agreement. 

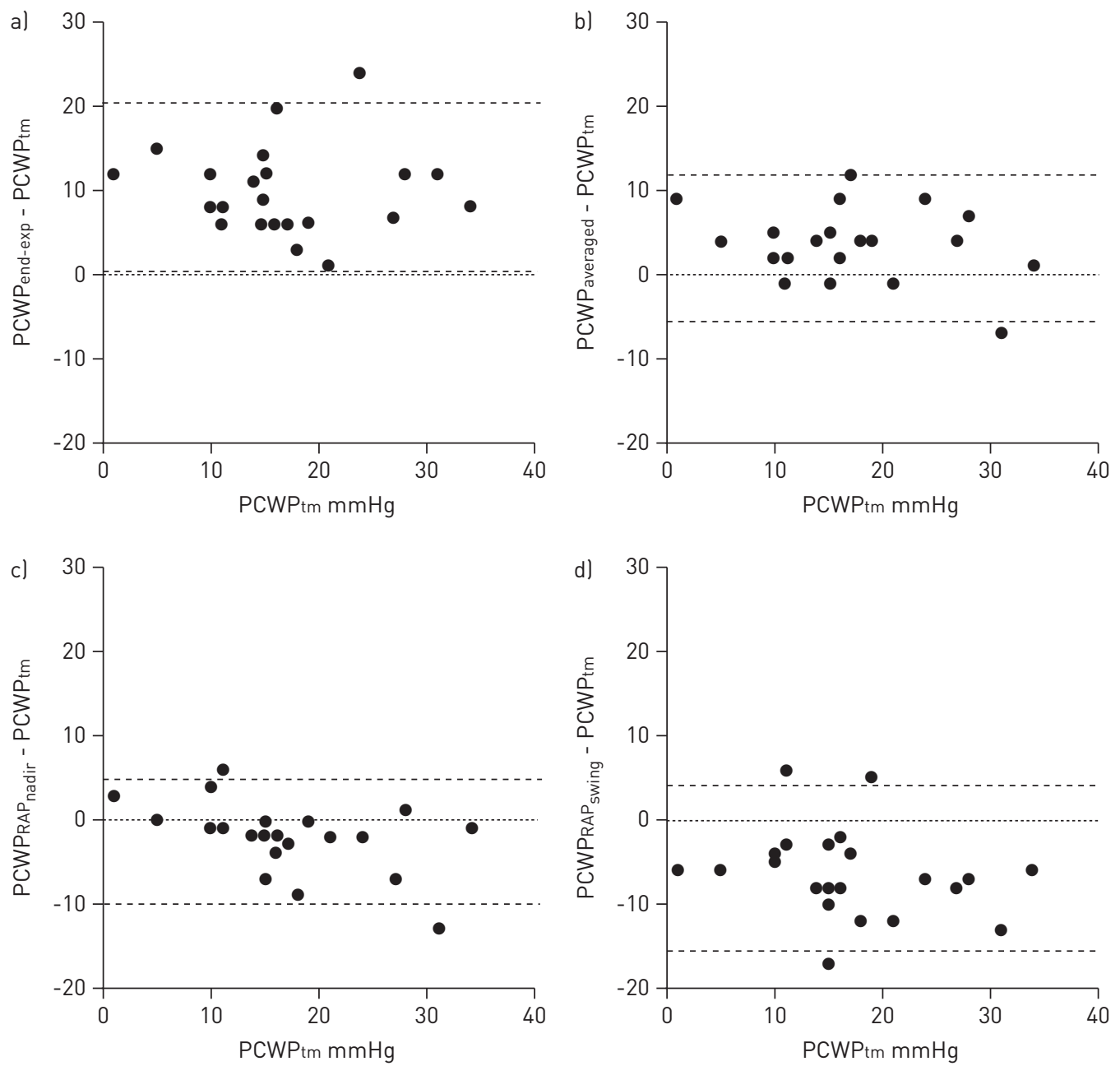

FIGURE 5 Bland-Altman analyses of the difference between pulmonary capillary wedge pressure (PCWP) and transmural pulmonary capillary wedge pressure (PCWPtm) plotted versus the PCWPtm. a) PCWP measured at the end of expiration (PCWPend-exp); b) PCWP averaged over the respiratory cycle (PCWPaveraged); c) PCWP corrected with the lowest point of RAP during expiration (PCWPRAP ${ }_{\text {nadir }}$ ); and d) PCWP corrected with the swing in RAP (PCWPRAP swing $)$. Dotted lines represent the $95 \%$ limits of agreements.

plots of the four methods of mPAP and PCWP measurements during exercise are shown in figures 4 and 5, respectively, and summarised in table 3 .

Effect of intrathoracic pressure swings on PCWP, MPAP and RAP

See table 2 for a summary of the pressure fluctuations. Figure 6 illustrates the relationship between the swing in mPAP (mPAPswing) and PCWP (PCWPswing). The mPAPswing and PCWPswing did not differ (mean difference $0.9 \mathrm{mmHg}, \mathrm{p}=0.35$ ). There was a strict relationship between RAPswing and PCWPswing $\left(\mathrm{r}^{2}=0.9, \mathrm{p}<0.001\right)$, with a slope of 1.02 , and no significant difference between RAPswing and PCWPswing (mean difference $0.7 \mathrm{mmHg}, \mathrm{p}=0.28$ ).

\section{Discussion}

Errors due to respiratory variation are a major concern when interpreting central vascular pressures during exercise, especially in patients with airflow limitation. The aim of this study was to evaluate the potential error introduced in mPAP by measuring at the end of expiration only and to evaluate potential correction methods. We found that: 1) a significant error is introduced when MPAP and PCWP are measured at the end of expiration in exercising COPD patients, due to increases in expiratory intrathoracic pressure; 2) averaging mPAP and PCWP over the respiratory cycle are better estimates of mPAPtm and PCWPtm; 3 ) the right atrial waveform can be used to correct for intrathoracic pressure in patients without right heart failure; 4) the transpulmonary pressure gradient (mPAP - PCWP) and, thus, PVR, is unaffected by swings in intrathoracic pressure. To better understand individual patients, pulmonary haemodynamics and 
TABLE 3 Summary of Bland-Altman analyses performed

Subjects $n$

\begin{tabular}{ll}
\hline mPAPend-exp & 3 \\
mPAPaveraged & 30 \\
mPAPRAP $_{\text {nadir }}$ & 3 \\
mPAPRAPswing & 3 \\
PCWPend-exp & 2 \\
PCWPaveraged & 2 \\
PCWPRAPnadir & 2 \\
PCWPRAPswing & 22
\end{tabular}

$\mathbf{r}^{2}$

0.86
0.92
0.94
0.86
0.69
0.75
0.73
0.64

Bias $\mathrm{mmHg}$

$10.3+5.9$

$2.5 \pm 4.4$

$-3.6 \pm 3.8$

$-9.3 \pm 5.9$

$9.9 \pm 5.3$

$3.2 \pm 4.4$

$-2.0 \pm 4.2$

$-6.3 \pm 5.3$
$95 \%$ limits

of agreement $\mathrm{mmHg}$

Data are presented as mean $\pm S D$, unless otherwise stated. mPAP: mean pulmonary artery pressure; mPAPend-exp: mPAP measured at the end of expiration; mPAPaveraged: MPAP averaged over the respiratory cycle; mPAPRAP nadir: MPAP corrected with the lowest point of right atrial pressure (RAP); PPAPRAP $_{\text {swing }}$ : mPAP corrected with the swing in RAP; PCWP: pulmonary capillary wedge pressure; PCWPend-exp: PCWP measured at endexpiration; PCWPaveraged: PCWP averaged over the respiratory cycle; PCWPRAP nadir: PCWP corrected for the lowest point of RAP; PCWPRAP swing: PCWP corrected for the swing in RAP.

mechanics should be reported (averaged over several cycles) at the end of expiration, the end of inspiration and averaged over the respiratory cycle raw and corrected for RAPnadir in routine clinical practice.

Our findings support the use of mPAP and PCWP averaged over two or three respiratory cycles in order to acquire more accurate assessment of the mPAPtm and PCWPtm during exercise in COPD patients. The patients in the present study showed a wide range of expiratory Poes at exercise, reaching as much as $25 \mathrm{mmHg}$, which is consistent with previous studies on pulmonary mechanics [20,21]. In these studies, as well as in the present study, the positive excursion of Poes during expiration is at least as large as the negative excursion during inspiration. It is, therefore, not surprising that mPAP averaged over the respiratory cycle is a more realistic measure of intravascular pressure. Although more accurate than mPAPend-exp, mPAPaveraged was still a slight overestimation, which can be explained by the increased expiratory time. In COPD patients, expiratory time during exercise is longer than inspiratory time [22]. As a consequence, mPAPaveraged is more influenced by the "high" expiratory mPAP than by the "low" inspiratory MPAP.

The usefulness of the RAP waveform to estimate the pressure surrounding the heart was shown by TYBERG et al. [16]. This method assumes that pressure in the very compliant right atrium is predominantly dependent on pressure surrounding the heart (pericardial pressure or, in this case, intrathoracic pressure), rather than by right atrial volume. We showed that this method was useful during exercise in COPD patients, as long as RAPnadir during expiration was used. This is explained by the fact that during the right atrial contraction, a dissociation between RAP and intrathoracic pressure is created. Therefore, only the pressure of an empty and relaxing right atrium is useful to estimate intrathoracic pressure. We found a small bias with mPAPtm and PCWPtm when RAPnadir was used to correct expiratory mPAP and PCWP, with a very reasonable $95 \%$ limits of agreement. The small overcorrection in all patients is because it is

FIGURE 6 The relationship between the swing in mean pulmonary artery pressure (mPAPswing) and the swing in pulmonary capillary wedge pressure (PCWPswing).

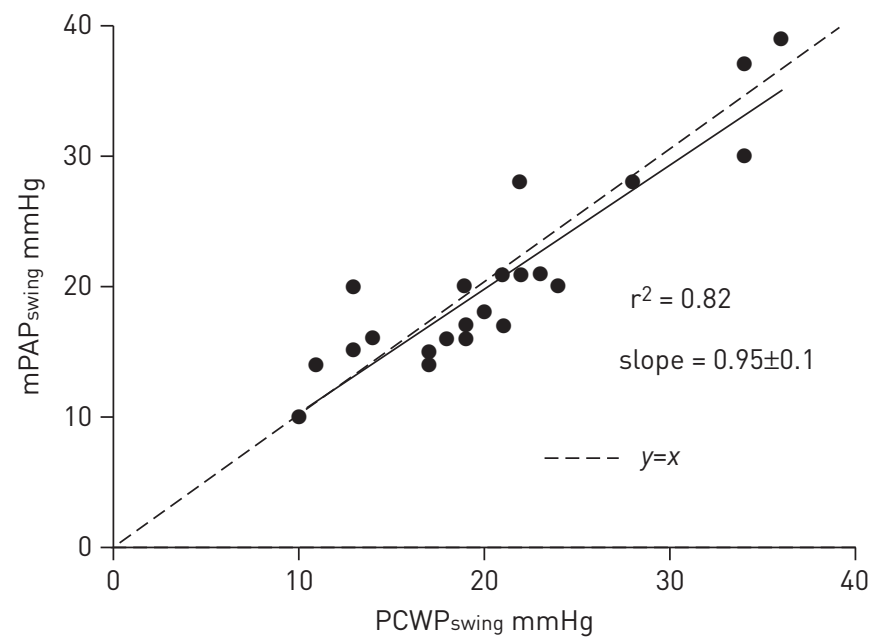


unlikely that RAPnadir can be lower than intrathoracic pressure at the same moment. This method may not be useful in patients with more pronounced right heart failure, as this causes RAP to rise, even during relaxation. Despite the average $\mathrm{MPAP}$ at rest in our cohort of COPD patients being higher than normally reported in COPD $[15,23]$, only two patients showed a RAPnadir higher than intrathoracic pressure during exercise. We previously showed that some patients with COPD have impaired venous return due to the high expiratory intrathoracic pressure, which brings RAPnadir even closer to intrathoracic pressure. Furthermore, we showed that RAPswing to estimate expiratory intrathoracic pressure was not useful in our patients. As during inspiration, RAP can reach significant negative values, the total swing is larger than the positive excursion of intrathoracic pressure with expiration. Correction of $\mathrm{mPAP}$ with RAPswing therefore leads to an underestimation of mPAPtm.

Lastly, we showed that the swings in MPAP, PCWP and RAP were similar. This has several convenient implications. The consequence of an identical effect of intrathoracic pressure swing on MPAP and PCWP is that the difference between the two, the transpulmonary pressure gradient, is unaffected by the swing in intrathoracic pressure. This only holds when both the MPAP and PCWP are recorded at the same time point in the respiratory cycle. So, although individually, $\mathrm{mPAP}$ and PCWP are overestimations of intravascular pressure, the mPAPend-exp and PCWPend-exp combined lead to the correct transpulmonary pressure gradient or PVR (transpulmonary gradient/cardiac output). It underscores the importance of PVR as part of the suggested definition of exercise induced pulmonary arterial hypertension [2], as it prevents patients being diagnosed simply because of an increased intrathoracic pressure. The similar rise in mPAP and PCWP from inspiration to expiration is consistent with the high intrathoracic pressure per se not contributing to right ventricular afterload, which is in agreement with previous literature on the effect of positive end expiratory pressure on right ventricular afterload [24, 25].

The similar effect of intrathoracic pressure swings in RAP and PCWP also has a potential implication in evaluating exercise haemodynamics. The increase in PCWP calculated as a ratio to the increase in RAP, as previously suggested [26], is unaffected by intrathoracic pressure swings. This ratio might therefore be of potential help in the difficult situation of a high PCWP with exercise in the presence of intrathoracic pressure swings, a situation that is common in COPD patients. This would be especially helpful in diagnosing exercise-induced heart failure with preserved ejection fraction [3].

The patients in the present study had at least moderate airflow limitation, probably a worst-case scenario for the influence of intrathoracic pressure on central pressure measurements. We can only speculate to what extent our findings can be extrapolated to patients with less severe airflow limitation or the normal elderly population. The positive expiratory intrathoracic pressure in COPD is mainly due to the use of expiratory muscles, which is not well related to the airflow limitation, and the pattern of expiratory muscle recruitment differs between patients [27]. In the healthy population, the use of expiratory muscles can lead to substantial positive intrathoracic pressure as well, albeit only at maximal exercise [28]. Pulmonary vascular pressure, therefore, should be averaged over two to three respiratory cycles, not only in COPD [7, 8, 29], but also healthy subjects [11-13]. Whether mPAPaveraged is a more accurate estimate of the intravascular pressure at maximal exercise in this population remains unknown, and depends on the amplitude and the length of the inspiratory and expiratory excursions in intrathoracic pressure. What is clear is that respiratory variation in central haemodynamic pressures during maximal exercise is not only present in severe COPD.

Limitations of this study warrant discussion. The average mPAP at rest in our cohort of COPD patients is higher than normally reported in COPD due to a referral bias, as our hospital is a pulmonary hypertension centre. Therefore, no conclusion should be drawn with regards to the incidence and severity of pulmonary hypertension at rest or during exercise in COPD from this study. We felt it was justified to use mPAP and PCWP after subtracting Poes as a gold standard, although small errors might be present in the measurement of Poes. This was the only method in which intrathoracic pressure was taken into account when evaluating mPAP and PCWP, and therefore the best way to answer our question.

In conclusion, the present study shows that substantial errors are introduced in the interpretation of the absolute values of mPAP and PCWP during exercise in COPD when read only at the end of expiration. In order to acquire more accurate values, averaging over the respiratory cycle or correction with the estimated intrathoracic pressure from the RAP waveform should be performed. The transpulmonary gradient is unaffected by the respiratory swings when its components are measured at the same time point in the respiratory cycle.

\section{References}

Saggar R, Khanna D, Shapiro S, et al. Effect of ambrisentan treatment on exercise-induced pulmonary hypertension in systemic sclerosis: a prospective single-center, open-label pilot study. Arthritis Rheum 2012; 64: 4072-4077.

2 Tolle JJ, Waxman AB, Van Horn TL, et al. Exercise-induced pulmonary arterial hypertension. Circulation 2008; 118: $2183-2189$. 
3 Borlaug BA, Nishimura RA, Sorajja P, et al. Exercise hemodynamics enhance diagnosis of early heart failure with preserved ejection fraction. Circ Heart Fail 2010; 3: 588-595.

4 Burrows B, Kettel LJ, Niden AH, et al. Patterns of cardiovascular dysfunction in chronic obstructive lung disease. N Engl J Med 1972; 286: 912-918.

5 Macchia A, Rodriguez Moncalvo JJ, Kleinert M, et al. Unrecognised ventricular dysfunction in COPD. Eur Respir J 2012; 39: 51-58.

6 Champion HC, Michelakis ED, Hassoun PM. Comprehensive invasive and noninvasive approach to the right ventricle-pulmonary circulation unit: state of the art and clinical and research implications. Circulation 2009; 120: 992-1007.

7 Lockhart A, Tzareva M, Nader F, et al. Elevated pulmonary artery wedge pressure at rest and during exercise in chronic bronchitis: fact or fancy. Clin Sci 1969; 37: 503-517.

8 Butler J, Schrijen F, Henriquez A, et al. Cause of the raised wedge pressure on exercise in chronic obstructive pulmonary disease. Am Rev Respir Dis 1988; 138: 350-354.

9 Light RW, Mintz HM, Linden GS, et al. Hemodynamics of patients with severe chronic obstructive pulmonary disease during progressive upright exercise. Am Rev Respir Dis 1984; 130: 391-395.

10 Mahler DA, Brent BN, Loke J, et al. Right ventricular performance and central circulatory hemodynamics during upright exercise in patients with chronic obstructive pulmonary disease. Am Rev Respir Dis 1984; 130: 722-729.

11 Granath A, Jonsson B, Strandell T. Circulation in healthy old men, studied by right heart catheterization at rest and during exercise in supine and sitting position. Acta Med Scand 1964; 176: 425-446.

12 Groves BM, Reeves JT, Sutton JR, et al. Operation Everest II: elevated high-altitude pulmonary resistance unresponsive to oxygen. J Appl Physiol 1987; 63: 521-530.

13 Hickam JB, Cargill WH. Effect of exercise on cardiac output and pulmonary arterial pressure in normal persons and in patients with cardiovascular disease and pulmonary emphysema. J Clin Invest 1948; 27: 10-23.

14 Blanco I, Gimeno E, Munoz PA, et al. Hemodynamic and gas exchange effects of sildenafil in patients with chronic obstructive pulmonary disease and pulmonary hypertension. Am J Respir Crit Care Med 2010; 181: 270-278.

15 Mykland Hilde J, Skjørten I, Hansteen V, et al. Hemodynamic responses to exercise in patients with COPD. Eur Respir J 2013; 41: 1031-1041.

16 Tyberg JV, Taichman GC, Smith ER, et al. The relationship between pericardial pressure and right atrial pressure: an intraoperative study. Circulation 1986; 73: 428-432.

17 Macintyre N, Crapo RO, Viegi G, et al. Standardisation of the single-breath determination of carbon monoxide uptake in the lung. Eur Respir J 2005; 26: 720-735.

18 Wanger J, Clausen JL, Coates A, et al. Standardisation of the measurement of lung volumes. Eur Respir J 2005; 26 : 511-522.

19 Milic-Emili J, Mead J, Turner JM, et al. Improved technique for estimating pleural pressure from esophageal balloons. J Appl Physiol 1964; 19: 207-211.

20 Laveneziana P, Webb KA, Ora J, et al. Evolution of dyspnea during exercise in chronic obstructive pulmonary disease: impact of critical volume constraints. Am J Respir Crit Care Med 2011; 184: 1367-1373.

21 Potter WA, Olafsson S, Hyatt RE. Ventilatory mechanics and expiratory flow limitation during exercise in patients with obstructive lung disease. J Clin Invest 1971; 50: 910-919.

22 Wilkens $\mathrm{H}$, Weingard B, Lo Mauro A, et al. Breathing pattern and chest wall volumes during exercise in patients with cystic fibrosis, pulmonary fibrosis and COPD before and after lung transplantation. Thorax 2010; 65: 808-814. Chaouat A, Naeije R, Weitzenblum E. Pulmonary hypertension in COPD. Eur Respir J 2008; 32: 1371-1385.

Luecke T, Pelosi P. Clinical review: positive end-expiratory pressure and cardiac output. Crit Care 2005; 9: 607-621. Pinsky MR. Cardiovascular issues in respiratory care. Chest 2005; 128: 592S-597S.

Reeves JT, Groves BM, Cymerman A, et al. Operation Everest II: cardiac filling pressures during cycle exercise at sea level. Respir Physiol 1990; 80: 147-154.

27 Louvaris Z, Zakynthinos S, Aliverti A, et al. Heliox increases quadriceps muscle oxygen delivery during exercise in COPD patients with and without dynamic hyperinflation. J Appl Physiol 2010; 113: 1012-1023.

28 Kearon MC, Summers E, Jones NL, et al. Breathing during prolonged exercise in humans. J Physiol 1991; 442: 477-487.

29 Rice DL, Awe RJ, Gaasch WH, et al. Wedge pressure measurement in obstructive pulmonary disease. Chest 1974; 66: 628-632. 